\title{
Improvement of endothelial function following initiation of testosterone replacement therapy
}

\author{
Daniel A. Shoskes, Barbara Tucky, Allan S. Polackwich \\ Glickman Urological and Kidney Institute, Cleveland Clinic, Cleveland, OH 44195, USA \\ Contributions: (I) Conception and design: DA Shoskes, AS Polackwich; (II) Administrative support: DA Shoskes, B Tucky; (III) Provision of study \\ materials or patients: DA Shoskes, AS Polackwich; (IV) Collection and assembly of data: DA Shoskes, B Tucky; (V) Data analysis and interpretation: \\ DA Shoskes, AS Polackwich; (VI) Manuscript writing: All authors; (VII) Final approval of manuscript: All authors. \\ Correspondence to: Daniel A. Shoskes, M.D. Glickman Urological and Kidney Institute, Cleveland Clinic, 9500 Euclid Avenue, Desk Q10-1, \\ Cleveland, OH 44195, USA. Email: dshoskes@gmail.com.
}

Background: Isolated recent studies have suggested an increased risk of heart attack as early as 3 months
following testosterone replacement therapy (TRT). Such a rapid risk increase would likely require rapid
deterioration of arterial endothelial function. Our goal was to assess arterial endothelial function in
hypogonadal men prior to and at least 3 months after initiation of TRT. Methods: Adult men were consented if they had symptoms of hypogonadism, a total testosterone $<350 \mathrm{ng} / \mathrm{dL}$, and planned to begin TRT. Endothelial function was non-invasively assessed using the EndoPAT-2000 machine. We measured the augmentation index (AI) (normal $<3 \%$ ), a measure of arterial stiffness and reactive hyperemia index (RHI), a measure of endothelial vasodilation (normal $>1.69$ ). Prior studies suggest that a $10 \%$ level of day-to-day test variability is expected. Endothelial function was reassessed at the next clinic visit, between 3 and 6 months if the patients were compliant with therapy. Changes in continuous variables were assessed with the paired $t$ test.

Results: Twenty-three patients were consented with a mean age of 52.7 years (range, 34-68 years) and starting testosterone $196.9 \mathrm{ng} / \mathrm{dL}$ (range, 35-339 $\mathrm{ng} / \mathrm{dL}$ ). There was a history of diabetes in four, hypertension in ten and coronary artery disease in five. Mean RHI was $1.67 \pm 0.37(70 \%$ were abnormal) and mean AI was $2.57 \% \pm 14.0 \%$ (39\% were abnormal). There were no cardiac events. At follow-up 20 patients were compliant with therapy and retested. Mean testosterone increased from 203 to $511(\mathrm{P}<0.0001)$. Mean RHI improved from 1.70 to $2.14(\mathrm{P}=0.01)$. Mean $\mathrm{AI}$ improved from $2.9 \%$ to $-1.75 \%(\mathrm{P}=0.01)$. In four men RHI worsened but in each case less than the $10 \%$ error of the test. No man had worsened AI.

Conclusions: Men with symptomatic hypogonadism often have abnormal arterial endothelial function. Following TRT, endothelial function either remains unchanged or improves.

Keywords: Testosterone; endothelial function; cardiovascular

Submitted Jun 02, 2016. Accepted for publication Jun 15, 2016.

doi: 10.21037/tau.2016.08.04

View this article at: http://dx.doi.org/10.21037/tau.2016.08.04

\section{Introduction}

The cardiac risk of starting testosterone replacement therapy (TRT) is controversial. Three recent studies which suggested an increase risk of cardiovascular events following TRT (1-3) led to an FDA review and updated labeling that indicates a possible increased risk of heart attacks and strokes following TRT. The methodology and conclusions of these studies have been criticized (4) and position statements and guidelines from medical societies do not support the FDA's concerns (5). Nevertheless, concern remains whether TRT can be safely given to men, especially those with cardiac risk factors.

If one accepts the hypothesis that TRT leads to early cardiovascular complications, there must be a mechanism 
that would lead to myocardial infarction in as early as 3 months. Since TRT does not cause progression of atherosclerotic plaque (6), the more likely mechanism in this short timeframe would be through worsening of endothelial function (7). Furthermore, since cardiovascular events are relatively rare following TRT, if endothelial dysfunction were indeed the mechanism linking the two, one would expect a higher incidence of endothelial dysfunction than myocardial infarction. Because of this increased incidence, this may provide a useful surrogate endpoint to more effectively evaluate the effect of TRT on cardiovascular function.

Endothelial cell dysfunction can be measured by assessing peripheral arterial tone and it's response to nitric oxide mediated relaxation induced by ischemia-reperfusion and sheer stress. The EndoPAT-2000 (Itamar Medical, Caesarea, Israel) is a device that non-invasively measures vascular endothelial cell function and arterial stiffness by measuring the peripheral arterial tone of the index finger before and after temporary occlusion of flow with a blood pressure cuff (8). If indeed TRT leads to increased early cardiac events we hypothesize that institution of TRT would worsen arterial endothelial function as measured by EndoPAT.

\section{Methods}

Adult men were consented for this IRB approved study from our Men's Health clinic if they had symptoms of hypogonadism, a total testosterone $<350 \mathrm{ng} / \mathrm{dL}$, and planned to begin TRT. Demographic data was collected including medical comorbidities and starting total testosterone values. The first EndoPAT test was done before starting TRT and the second between 3-6 months later at the first follow-up appointment if the patient was compliant with therapy. Topical gels or Testopel were used for TRT and target testosterone confirmed post therapy.

Men were tested with an EndoPAT-2000 machine according to manufacturer's instructions. Briefly, men sat in a quiet room with a device on both index fingers that measures arterial tone. A blood pressure cuff on the left arm occluded arterial blood flow for 5 minutes and then released. The software then calculated the augmentation index (AI), a measure of arterial stiffness and reactive hyperemia index (RHI), a measure of nitric oxide mediated endothelial vasodilation. Prior studies suggest that a $10 \%$ level of day-to-day test variability is expected (9). Both elevated AI and diminished RHI suggest vascular and endothelial disease.

Changes in continuous variables were assessed with the paired $t$-test and significance set at $\mathrm{P}<0.05$. Statistical analyses were performed with Prism 5.0 for Macintosh (Graphpad software).

\section{Results}

Twenty-three patients were consented with a mean age of 52.7 years (range, 34-68 years). Mean starting testosterone $196.9 \mathrm{ng} / \mathrm{dL}$ (range, 35-339 ng/dL). There was a selfreported history of diabetes in four men, hypertension in ten and coronary artery disease in five. At baseline measurement mean RHI was $1.67 \pm 0.37$ (70\% were abnormal) and mean AI was $2.57 \% \pm 14.0 \%$ (39\% were abnormal). No patient subsequently developed a clinical cardiac event.

At first clinical follow-up 3-6 months later, 20 patients were compliant with therapy and retested. Mean total testosterone increased from 203 to $511 \mathrm{ng} / \mathrm{dL}(\mathrm{P}<0.0001)$ (Figure 1). Mean RHI improved from 1.70 to $2.14(\mathrm{P}=0.01)$ (Figure 2). Mean AI improved from $2.9 \%$ to $-1.75 \%$ $(\mathrm{P}=0.01)$ (Figure 3). In four men RHI was lower than before starting TRT but in each case the decrease was less than the $10 \%$ error of the test (range, $1.3-6.3 \%$ ). No man had worsening of AI.

\section{Discussion}

The cardiovascular risks of TRT remain controversial. Concern initially developed from cardiac outcomes in studies of women. The Women's Health Initiative found a modest increase in cardiovascular events with hormonal replacement; seven additional cardiac heart disease events and eight additional strokes per 10,000 person years (10).

Following this, a series of studies raised the possibility that TRT might also increase the risk of cardiovascular complications including stroke and myocardial infarction (3) and other cardiac events (1). There have been criticisms to the design of these studies (11), especially since they required significant statistical adjustment to arrive at their conclusions. Additionally these findings were counterintuitive given that TRT is known to improve several risk factors for cardiovascular disease including fasting glucose and waist circumference (12). Indeed, subsequent studies have shown a reduced risk of myocardial infarction following TRT, this includes a recent study of 83,010 men in a VA study, one of the largest studies on this topic to date $(1,13)$. Nevertheless, the FDA recently 


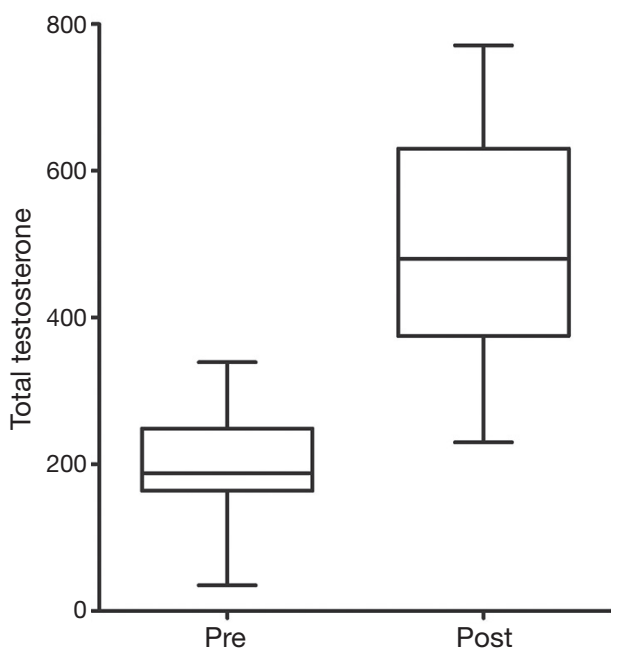

Figure 1 Box plot of total testosterone values before and after therapy $(\mathrm{P}<0.0001)$.

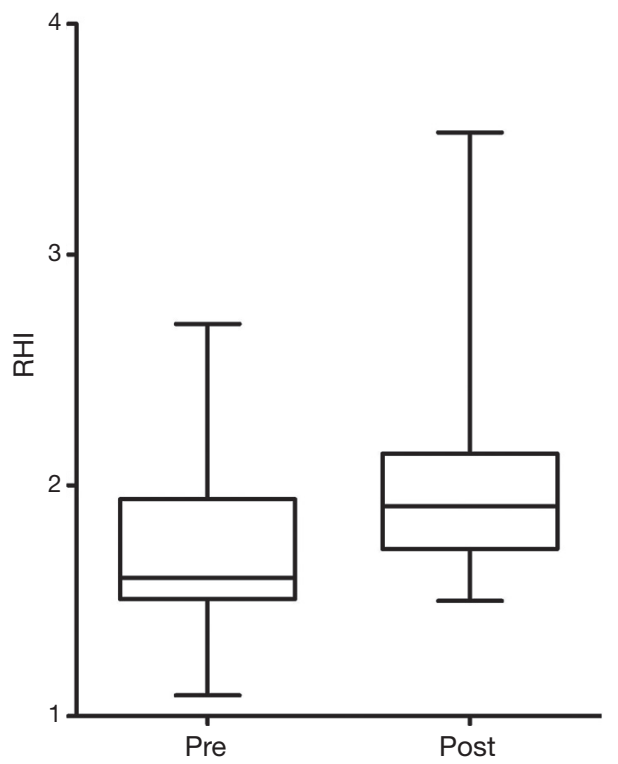

Figure 2 Comparison of reactive hyperemic index (RHI) before and after testosterone therapy. Higher RHI means better endothelial function $(\mathrm{P}=0.01)$.

required a labeling change to inform patients of a possible increased risk of heart attack and stroke (http://www.fda. gov/Safety/MedWatch/SafetyInformation/SafetyAlertsforH umanMedicalProducts/ucm436280.htm).

Part of this controversy is due to the fact that no definite mechanism for testosterone's effect on cardiovascular disease has been discovered. Certainly polycythemia is a

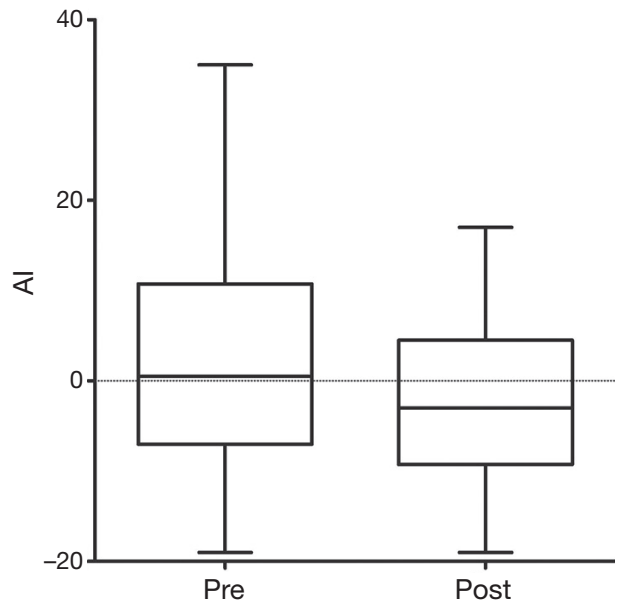

Figure 3 Comparison of augmentation index (AI) before and after testosterone therapy. Lower AI means less arterial stiffness $(\mathrm{P}=0.01)$.

possible side effect that can promote thrombosis and fluid retention which can exacerbate severe heart failure, but monitoring for polycythemia is a standard as part of treating patients with testosterone and should be limited in most patients. Atherosclerosis and other traditional risk factors for heart disease are too slow for the time scale suggested by the studies that demonstrate an increase in cardiac events. If indeed TRT increases cardiovascular risk, at least in a subset of patients, we believe an effective surrogate marker would be endothelial dysfunction, especially since cardiovascular events occur so rarely. While this is not a proven mechanism of testosterone's effect on cardiovascular health, we propose that it is one of the most likely mechanisms due to the time course of the proposed increase in cardiovascular events. Abnormal endothelial function as measured by peripheral arterial tone is known to be predictive of late cardiovascular events (14). Indeed, hypogonadism has already been associated with endothelial dysfunction and cardiovascular risk in men with renal disease (15). The EndoPAT machine used in this study uses changes in peripheral arterial tone before and after brachial artery occlusion to produce two values: RHI which measures nitric oxide mediated endothelial relaxation (higher is better) and AI which measures arterial stiffness (lower is better) (8). While few studies have followed patients longitudinally, between day variability of RHI is about $10 \%$ (9).

In our study, both RHI and AI either stayed the same or improved in all patients 3-6 months after starting TRT. As expected, most of the patients with hypogonadism started 
out with abnormal values (70\% abnormal RHI and 39\% abnormal $\mathrm{AI}$ ), confirming a link between hypogonadism and cardiovascular risk (16). This supports previous data from other studies that demonstrates patients with hypogonadism have increased rates cardiovascular events and death (17). That endothelial function did not worsen after 3-6 months of therapy does not necessarily suggest ongoing improvement; however the key studies implicating TRT as a cause of increased risk for cardiovascular complications noted those complications as early as 3 months into therapy. Our study shows that endothelial dysfunction, a possible mechanism for such changes does not change in this time period, adding to the data that testosterone therapy does not worsen cardiac function in the short-term.

This represents the first study evaluating the effects of testosterone on endothelial dysfunction as measured by EndoPAT, a proposed mechanism for testosterone's yet unclear effect on cardiovascular health. Limitations of our study include its small size. Despite this, its prospective nature and low number of patients lost to follow-up reinforce the importance of the findings.

\section{Conclusions}

We found that endothelial dysfunction does not worsen in patients starting testosterone therapy within the first 3-6 months of therapy. While the full effects of testosterone therapy on the cardiovascular system have not been fully elucidated, this study increases the body of literature that it is safe for use within patients for whom it is clearly indicated.

\section{Acknowledgements}

None.

\section{Footnote}

Conflicts of Interest: The authors have no conflicts of interest to declare.

Ethical Statement: The study was approved by IRB of \#14706 and written informed consent was obtained from all patients.

\section{References}

1. Basaria S, Coviello AD, Travison TG, et al. Adverse events associated with testosterone administration. $\mathrm{N}$ Engl J Med 2010;363:109-22.

2. Finkle WD, Greenland S, Ridgeway GK, et al. Increased risk of non-fatal myocardial infarction following testosterone therapy prescription in men. PLoS One 2014;9:e85805.

3. Vigen R, O'Donnell CI, Barón AE, et al. Association of testosterone therapy with mortality, myocardial infarction, and stroke in men with low testosterone levels. JAMA 2013;310:1829-36.

4. Morgentaler A. Testosterone, cardiovascular risk, and hormonophobia. J Sex Med 2014;11:1362-6.

5. Goodman N, Guay A, Dandona P, et al. American association of clinical endocrinologists and american college of endocrinology position statement on the association of testosterone and cardiovascular risk. Endocr Pract 2015;21:1066-73.

6. Basaria S, Harman SM, Travison TG, et al. Effects of Testosterone Administration for 3 Years on Subclinical Atherosclerosis Progression in Older Men With Low or Low-Normal Testosterone Levels: A Randomized Clinical Trial. JAMA 2015;314:570-81.

7. Tabata N, Hokimoto S, Akasaka T, et al. Differential impact of peripheral endothelial dysfunction on subsequent cardiovascular events following percutaneous coronary intervention between chronic kidney disease (CKD) and non-CKD patients. Heart Vessels 2016;31:1038-44.

8. Faizi AK, Kornmo DW, Agewall S. Evaluation of endothelial function using finger plethysmography. Clin Physiol Funct Imaging 2009;29:372-5.

9. Onkelinx S, Cornelissen V, Goetschalckx K, et al. Reproducibility of different methods to measure the endothelial function. Vasc Med 2012;17:79-84.

10. Rossouw JE, Anderson GL, Prentice RL, et al. Risks and benefits of estrogen plus progestin in healthy postmenopausal women: principal results From the Women's Health Initiative randomized controlled trial. JAMA 2002;288:321-33.

11. Traish AM, Guay AT, Morgentaler A. Death by testosterone? We think not! J Sex Med 2014;11:624-9.

12. Aversa A, Bruzziches R, Francomano D, et al. Effects of testosterone undecanoate on cardiovascular risk factors and atherosclerosis in middle-aged men with late-onset hypogonadism and metabolic syndrome: results from a 24-month, randomized, double-blind, placebo-controlled study. J Sex Med 2010;7:3495-503.

13. Sharma R, Oni OA, Gupta K, et al. Normalization of testosterone level is associated with reduced incidence of 
myocardial infarction and mortality in men. Eur Heart J 2015;36:2706-15.

14. Rubinshtein R, Kuvin JT, Soffler M, et al. Assessment of endothelial function by non-invasive peripheral arterial tonometry predicts late cardiovascular adverse events. Eur Heart J 2010;31:1142-8.

15. Yilmaz MI, Sonmez A, Qureshi AR, et al. Endogenous testosterone, endothelial dysfunction, and cardiovascular events in men with nondialysis chronic kidney disease.

Cite this article as: Shoskes DA, Tucky B, Polackwich AS. Improvement of endothelial function following initiation of testosterone replacement therapy. Transl Androl Urol 2016;5(6):819-823. doi: 10.21037/tau.2016.08.04
Clin J Am Soc Nephrol 2011;6:1617-25.

16. Corona G, Rastrelli G, Monami M, et al. Hypogonadism as a risk factor for cardiovascular mortality in men: a metaanalytic study. Eur J Endocrinol 2011;165:687-701.

17. Hyde Z, Norman PE, Flicker L, et al. Low free testosterone predicts mortality from cardiovascular disease but not other causes: the Health in Men Study. J Clin Endocrinol Metab 2012;97:179-89. 